\title{
STAT4 gene polymorphism in patients after renal allograft transplantation
}

\author{
EWA DAZBROWSKA-ŻAMOJCIN ${ }^{1}$, VIOLETTA DZIEDZIEJKO', KRZYSZTOF SAFRANOW', \\ LESZEK DOMAŃSKI ${ }^{3}$, SYLWIA SŁUCZANOWSKA-GEABOWSKA4, ANDRZEJ PAWLIK ${ }^{4}$ \\ ${ }^{1}$ Department of Pharmacology, Pomeranian Medical University, Szczecin, Poland \\ ${ }^{2}$ Department of Biochemistry and Medical Chemistry, Pomeranian Medical University, Szczecin, Poland \\ ${ }^{3}$ Department of Nephrology, Transplantology and Internal Medicine, Pomeranian Medical University, Szczecin, Poland \\ ${ }^{4}$ Department of Physiology, Pomeranian Medical University, Szczecin, Poland
}

\begin{abstract}
Introduction: STAT4 (signal transducer and activator of transcription 4) is involved in the regulation of innate and adaptive immune responses. Some studies have suggested that STAT4 may be involved in the immune response after graft transplantation. Several polymorphisms in the STAT4 gene have been identified. The most commonly studied polymorphism in the STAT4 gene is rs7574865. In our study, we examined whether this polymorphism is associated with the early and late functions of renal allografts.

Material and methods: A total of 270 recipients of first renal transplants were included in the study. Single nucleotide polymorphisms (SNPS) within the STAT4 gene were genotyped using TaqMan genotyping assays.

Results: There were no statistically significant associations between the STAT4 gene rs 7574865 polymorphism and delayed graft function, acute rejection, chronic allograft dysfunction, post-transplant diabetes mellitus, or creatinine serum concentrations after transplantation.

Conclusions: Our results suggest a lack of association between the STAT4 rs7574865 SNP and kidney allograft function in the Polish population.
\end{abstract}

Key words: STAT4, polymorphism, kidney, graft.

(Cent Eur J Immunol 2016; 41 (3): 255-259)

\section{Introduction}

STATs (signal transducers and activators of transcription) are DNA-binding transcription factors that induce the transcription of genes by recognising specific DNA sequences. There are seven different members of the STAT family (STAT1, STAT2, STAT3, STAT4, STAT5A, STAT5B, and STAT6), which are involved in the regulation of the innate and adaptive immune responses [1]. STAT4 protein comprises an N-terminal domain that plays an important role in phosphorylation and nuclear translocation, as well as a four-stranded helical coiled coil that is implicated in protein-protein interaction and nuclear import and export [2]. STAT4 plays a significant role in the regulation of natural killer (NK) cells, CD8+ $\mathrm{T}$ cells, and Th1 function, as well as in the differentiation of B cells and regulatory T (Treg) cells [3, 4]. STAT4 is expressed in activated peripheral blood monocytes, dendritic cells, and macrophages, and plays a significant role in the signalling pathway of several important cytokines $[5,6]$. Furthermore, dysregulation of STAT4 has been found in some autoimmune diseases [7-9].
STAT4 was mapped to chromosome $2 \mathrm{q} 32.2-\mathrm{q} 32.3$ [10]. The most commonly studied polymorphism in the STAT4 gene is rs $7574865[8,9,11]$. This polymorphism is located in the third intron of the STAT4 gene, but its functional consequences are not known. It is possible that the rs7574865 polymorphism influences STAT4 expression or phosphorylation. The STAT4 gene polymorphism is emerging as a novel risk factor for various diseases, in particular, autoimmune diseases, such as ulcerative colitis, rheumatoid arthritis, diabetes, systemic, lupus erythematosus, and Sjögren's syndrome [7-9, 11]. Some studies have suggested that STAT4 may be involved in the immune response after graft transplantation [12]. In our study, we examined whether STAT4 rs7574865 polymorphism is associated with the early and late functions of renal allografts.

\section{Material and methods}

A total of 270 recipients of first renal transplants were included in the study (166 males, 104 females, mean age $47.63 \pm 12.96$ years; transplantation performed between 2001 and 2009 in the Clinical Department of Nephrology

Correspondence: Andrzej Pawlik, PhD, Pomeranian Medical University, Powstańców Wielkopolskich 72, 70-111 Szczecin, Poland,

e-mail: pawand@poczta.onet.pl

Submitted: 05.04.2016; Accepted: 01.06.2016 
and Transplantology of the Pomeranian Medical University in Szczecin, Poland). The main causes of chronic kidney disease and renal transplantation were: glomerulonephritis (58\%), hypertension (9\%), and diabetes (9\%). The histories of the patients were analysed, taking into account delayed graft function (DGF), acute rejection, and chronic allograft dysfunction. Delayed graft function was defined as the need for haemodialysis within the first seven days after transplantation. Acute rejection episodes were defined by clinical diagnosis (elevated serum creatinine in the absence of other pathology, including infection, urinary tract obstruction, allograft artery stenosis, or cyclosporine toxicity) and were confirmed by positive biopsy. Chronic allograft dysfunction was diagnosed by eliminating other causes, including infections, urinary obstruction, allograft artery stenosis, or cyclosporine toxicity, and by changes in biopsy samples. This process was diagnosed clinically in patients that had a slow persistent rise in serum creatinine of at least $30 \%$ above baseline, usually accompanied by new or worsening hypertension and proteinuria (above $500 \mathrm{mg}$ / $24 \mathrm{~h}$ ). Anatomical problems were excluded by ultrasound and nuclear scans. Biopsy criteria included the presence of interstitial fibrosis, tubular atrophy, and, particularly, characteristic vascular changes, such as hypertrophy of the arterial intima and smooth muscle (intimal thickening) and glomerular sclerosis. All biopsies were reviewed by a renal pathologist, and the Banff working classification criteria were used in the histological classification of the biopsies [13]. Blood samples were collected from all patients for genetic analysis at the start of the study and for the evaluation of creatinine concentration at $1,3,6,12$, 24 , and 36 months after kidney transplantation. Creatinine concentration was measured using a colorimetric method. Patients with haemoglobin A1c continuously over 6.5\%, fasting blood glucose $\geq 7.0 \mathrm{mmol} / \mathrm{l}$, or requiring treatment with oral hypoglycaemic agents or insulin for more than three months after transplantation were diagnosed as having post-transplant diabetes mellitus (PTDM) [14].

All patients received a standard immunosuppressive protocol with triple-drug therapy, including calcineurin inhibitors (cyclosporine A in $75 \%$ and tacrolimus in $24 \%$ of recipients), azathioprine (55\%) or mycophenolate mofetil (37\%), and steroids (91\%). The study was approved by the Ethics Committee in Pomeranian Medical University, Szczecin, Poland, and written informed consent was obtained from all subjects.

\section{Genotyping}

SNPs within the STAT4 gene were genotyped using TaqMan genotyping assays. Genomic DNA was extracted from $200 \mu \mathrm{l}$ of whole blood samples using a GeneMATRIX Quick Blood DNA Purification Kit (EURx, Poland). Fluorescence data was captured using a 7500 FAST RealTime PCR System (Applied Biosystems, USA).

\section{Statistical analysis}

Chi-square test and Fisher's exact test were used to compare genotype and allele frequencies between groups. The serum concentrations of creatinine were compared between genotype groups using the non-parametric Kruskal-Wallis test because their distributions were significantly different from normal. A $p$-value $<0.05$ was considered statistically significant.

\section{Results}

The GG genotype was diagnosed in $58.15 \%$ of transplant recipients, GT in $34.44 \%$, and TT in $7.41 \%$. The distribution of the genotypes was in Hardy-Weinberg equilibrium $(p=0.25)$.

Delayed graft function was diagnosed in $34.39 \%$ of the patients with the GG genotype, $24.73 \%$ with GT, and

Table 1. The association between STAT4 genotypes and delayed graft function (DGF)

\begin{tabular}{|c|c|c|c|c|c|c|c|c|}
\hline & \multicolumn{2}{|c|}{ Without DGF } & \multicolumn{2}{|c|}{ DGF } & \multirow[t]{2}{*}{$p$-value ${ }^{\wedge}$} & & \multirow[t]{2}{*}{$p$-value* } & \multirow[t]{2}{*}{ OR $(95 \% \mathrm{CI})$} \\
\hline & $n$ & $\%$ & $n$ & $\%$ & & & & \\
\hline \multicolumn{9}{|c|}{ STAT4 rs7574865 genotype } \\
\hline GG & 103 & 65.61 & 54 & 34.39 & 0.20 & TT + GT vs. GG & 0.24 & $0.72(0.43-1.22)$ \\
\hline GT & 70 & 75.27 & 23 & 24.73 & & TT vs. GT + GG & 0.45 & $1.50(0.59-3.81)$ \\
\hline \multirow[t]{3}{*}{ TT } & 12 & 60.00 & 8 & 40.00 & & TT vs. GG & 0.63 & $1.27(0.49-3.30)$ \\
\hline & & & & & & GT vs. GG & 0.12 & $0.63(0.35-1.11)$ \\
\hline & & & & & & TT vs. GT & 0.18 & $2.03(0.74-5.58)$ \\
\hline \multicolumn{9}{|c|}{ STAT4 rs7574865 allele } \\
\hline $\mathrm{G}$ & 276 & 74.59 & 131 & 77.06 & & T vs. G & 0.59 & $0.87(0.57-1.34)$ \\
\hline $\mathrm{T}$ & 94 & 25.41 & 39 & 22.94 & & & & \\
\hline
\end{tabular}


Table 2. The association between STAT4 genotypes and acute rejection

\begin{tabular}{|c|c|c|c|c|c|c|c|c|}
\hline & \multicolumn{2}{|c|}{$\begin{array}{l}\text { Without acute } \\
\text { rejection }\end{array}$} & \multicolumn{2}{|c|}{ Acute rejection } & \multirow[t]{2}{*}{$p$-value $^{\wedge}$} & & \multirow[t]{2}{*}{$p$-value* } & \multirow[t]{2}{*}{ OR $(95 \% \mathrm{CI})$} \\
\hline & $n$ & $\%$ & $n$ & $\%$ & & & & \\
\hline \multicolumn{9}{|c|}{ STAT4 rs7574865 genotype } \\
\hline GG & 115 & 73.25 & 42 & 26.75 & 0.51 & TT + GT vs. GG & 0.78 & $0.90(0.52-1.57)$ \\
\hline GT & 68 & 73.12 & 25 & 26.88 & & TT vs. GT + GG & 0.30 & $0.48(0.14-1.70)$ \\
\hline \multirow[t]{3}{*}{ TT } & 17 & 85.00 & 3 & 15.00 & & TT vs. GG & 0.41 & $0.48(0.13-1.73)$ \\
\hline & & & & & & GT vs. GG & 1.00 & $1.01(0.56-1.80)$ \\
\hline & & & & & & TT vs. GT & 0.39 & $0.48(0.13-1.78)$ \\
\hline \multicolumn{9}{|c|}{ STAT4 rs7574865 allele } \\
\hline G & 298 & 74.50 & 109 & 77.86 & & T vs. G & 0.49 & $0.83(0.53-1.31)$ \\
\hline $\mathrm{T}$ & 102 & 25.50 & 31 & 22.14 & & & & \\
\hline
\end{tabular}

Table 3. The association between STAT4 genotypes and chronic allograft dysfunction

\begin{tabular}{|c|c|c|c|c|c|c|c|c|}
\hline & \multicolumn{2}{|c|}{$\begin{array}{c}\text { Without chronic } \\
\text { allograft dysfunction }\end{array}$} & \multicolumn{2}{|c|}{$\begin{array}{c}\text { Chronic allograft } \\
\text { dysfunction }\end{array}$} & \multirow[t]{2}{*}{$p$-value ${ }^{\wedge}$} & & \multirow[t]{2}{*}{$p$-value* } & \multirow[t]{2}{*}{ OR $(95 \%$ CI $)$} \\
\hline & $n$ & $\%$ & $n$ & $\%$ & & & & \\
\hline \multicolumn{9}{|c|}{ STAT4 rs7574865 genotype } \\
\hline GG & 123 & 78.34 & 34 & 21.66 & 0.43 & TT + GT vs. GG & 0.56 & $1.19(0.67-2.11)$ \\
\hline GT & 68 & 73.12 & 25 & 26.88 & & TT vs. GT + GG & 0.58 & $0.57(0.16-2.02)$ \\
\hline \multirow[t]{3}{*}{ TT } & 17 & 85.00 & 3 & 15.00 & & TT vs. GG & 0.77 & $0.64(0.18-2.31)$ \\
\hline & & & & & & GT vs. GG & 0.36 & $1.33(0.73-2.41)$ \\
\hline & & & & & & TT vs. GT & 0.39 & $0.48(0.13-1.78)$ \\
\hline \multicolumn{9}{|c|}{ STAT4 rs7574865 allele } \\
\hline G & 314 & 75.48 & 93 & 75.00 & & T vs. G & 0.91 & $1.03(0.65-1.63)$ \\
\hline $\mathrm{T}$ & 102 & 24.52 & 31 & 25.00 & & & & \\
\hline
\end{tabular}

Table 4. The association between STAT4 genotypes and post-transplant diabetes mellitus (PTDM)

\begin{tabular}{|c|c|c|c|c|c|c|c|c|}
\hline & \multicolumn{2}{|c|}{ PTDM absent } & \multicolumn{2}{|c|}{ PTDM present } & \multirow[t]{2}{*}{$p$-value ${ }^{\wedge}$} & & \multirow[t]{2}{*}{$p$-value* } & \multirow[t]{2}{*}{ OR $(95 \% \mathrm{CI})$} \\
\hline & $n$ & $\%$ & $n$ & $\%$ & & & & \\
\hline \multicolumn{9}{|c|}{ STAT4 rs7574865 genotype } \\
\hline GG & 81 & 84.38 & 15 & 15.63 & 0.67 & TT + GT vs. GG & 0.50 & $0.66(0.27-1.66)$ \\
\hline GT & 55 & 88.71 & 7 & 11.29 & & TT vs. GT + GG & 1.00 & $0.62(0.08-5.07)$ \\
\hline \multirow[t]{3}{*}{ TT } & 10 & 90.91 & 1 & 9.09 & & TT vs. GG & 1.00 & $0.54(0.06-4.54)$ \\
\hline & & & & & & GT vs. GG & 0.49 & $0.69(0.26-1.80)$ \\
\hline & & & & & & TT vs. GT & 1.00 & $0.79(0.09-7.10)$ \\
\hline \multicolumn{9}{|c|}{ STAT4 rs7574865 allele } \\
\hline G & 217 & 74.32 & 37 & 80.43 & & T vs. G & 0.46 & $0.70(0.32-1.53)$ \\
\hline $\mathrm{T}$ & 75 & 25.68 & 9 & 19.57 & & & & \\
\hline
\end{tabular}


Table 5. The association between STAT4 genotypes and serum creatinine after transplantation

\begin{tabular}{|c|c|c|c|c|c|c|c|}
\hline \multirow{3}{*}{$\begin{array}{l}\text { Creatinine } \\
\text { (mg/dl) }\end{array}$} & \multicolumn{6}{|c|}{ STAT4 rs7574865 genotype } & \multirow[t]{3}{*}{$p$-value ${ }^{\#}$} \\
\hline & \multicolumn{2}{|c|}{ GG } & \multicolumn{2}{|c|}{ GT } & \multicolumn{2}{|c|}{ TT } & \\
\hline & $n$ & Mean \pm SD & $n$ & Mean \pm SD & $n$ & Mean \pm SD & \\
\hline 1 month & 157 & $1.89 \pm 0.99$ & 93 & $1.84 \pm 0.72$ & 20 & $1.67 \pm 0.51$ & 0.81 \\
\hline 3 months & 157 & $1.77 \pm 0.61$ & 93 & $1.75 \pm 0.62$ & 20 & $1.76 \pm 0.52$ & 0.83 \\
\hline 6 months & 157 & $1.77 \pm 0.65$ & 92 & $1.81 \pm 0.67$ & 20 & $1.72 \pm 0.39$ & 0.93 \\
\hline 12 months & 153 & $1.76 \pm 0.59$ & 91 & $1.79 \pm 0.68$ & 20 & $1.67 \pm 0.45$ & 0.93 \\
\hline 24 months & 148 & $1.72 \pm 0.55$ & 90 & $1.79 \pm 0.69$ & 18 & $1.69 \pm 0.57$ & 0.85 \\
\hline 36 months & 139 & $1.73 \pm 0.55$ & 83 & $1.71 \pm 0.65$ & 18 & $1.75 \pm 0.79$ & 0.72 \\
\hline
\end{tabular}

$40.00 \%$ with TT. The differences were not statistically significant (Table 1).

Acute rejection was diagnosed in $26.75 \%$ of the carriers of the GG genotype, $26.88 \%$ of GT carriers, and $15.00 \%$ of those with TT. The differences were not statistically significant (Table 2).

Chronic allograft dysfunction was diagnosed in $21.66 \%$ of the patients with the GG genotype, $26.88 \%$ with GT, and $15.00 \%$ with TT. The differences were not statistically significant (Table 3 ).

Post-transplant diabetes mellitus was diagnosed in $15.63 \%$ of the patients with the GG genotype, $11.29 \%$ with GT, and $9.09 \%$ with TT. The differences were not statistically significant (Table 4).

Creatinine concentrations at 1, 3, 6, 12, 24, and 36 months after transplantation did not differ significantly between the different genotypes of the STAT4 gene rs7574865 polymorphism (Table 5).

\section{Discussion}

In this study we analysed the association between the STAT4 gene rs7574865 polymorphism and kidney allograft function. We examined the association between the STAT4 gene rs7574865 polymorphism and DGF, acute rejection, chronic allograft nephropathy, post-transplant diabetes, and creatinine concentrations up to 36 months after transplantation. There were no statistically significant associations between this polymorphism and early and late graft function.

STAT4 plays a crucial role in the function of innate and adaptive immune cells [15]. Moreover, dysregulated expression and aberrant activation of STAT4 is observed in many human autoimmune diseases. STAT4 also plays a significant role in the immune response after transplantation. STAT4 is critical in Th-cell activation. Chang et al. $[16,17]$ revealed that STAT4 deficiency prevented development of Th1 cells from post-transplant patients, and this correlated with reduced interferon $\gamma$ (IFN- $\gamma$ ) in Th1 cells.

Only a few studies have examined the role of STAT4 in the immune response after allograft transplantation.
Liang et al. analysed the various IFN- $\gamma$-dependent functions in terms of Th1 and Th2 responses during rejection. These authors investigated mice deficient in the transcription factors STAT4 and IFN- $\gamma$. The data showed that the STAT4-deficient groups have significantly prolonged graft survival [18].

Zhou et al. investigated the role of STAT4 signalling in allograft rejection and CTLA4-Ig-mediated tolerance [19]. STAT4(-/-) mice have impaired type 1 T-cell differentiation. The role of Th1 and Th2 cell differentiation in acute cardiac allograft rejection and in the induction of tolerance was examined in wild-type and STAT4(-/-) recipients. Analysis of in situ cytokine gene expression in the allografts confirmed decreased levels of IFN- $\gamma$ in STAT4(-/-) recipients. Blockade of the CD28/B7 co-stimulatory pathway prolonged cardiac graft survival in $100 \%$ of wild-type and STAT4(-/-) mice. These data suggest that the balance of Th1 and Th2 differentiation is not critical for acute rejection but does influence the tolerance induced by CD28/B7 blockade.

Eurich et al. evaluated the role of the STAT4 rs7574865 polymorphism in the development of $\mathrm{HCV}$-related graft disease based on protocol biopsies [20]. During an observation period, $46.5 \%$ of patients developed advanced fibrosis. Advanced fibrosis was observed significantly more frequently in patients with at least one T allele compared with homozygotes for the G allele. Significant differences in the duration of advanced fibrosis development were detected between patients with at least one T allele compared with the $\mathrm{G}$ allele. No impact was observed regarding the outcome of interferon-based antiviral treatment and the occurrence of acute cellular rejection. These results indicate a possible impact of the STAT4 T allele on graft fibrogenesis, thus explaining the significantly different graft behaviour observed after transplantation for $\mathrm{HCV}$-associated liver disease.

The STAT4 rs7574865 polymorphism has been correlated with diabetes susceptibility. Members of the STAT family are transcription factors that mediate the signalling events of many cytokines in immune and non-immune cells $[3,21]$. STAT4 is also involved in the development 
of a newly discovered subset of Th17 cells, which display a dominant role in autoimmunity-associated inflammation, including diabetes [22]. Results from recent studies have indicated an association between the rs 7574865 singlenucleotide polymorphism in the STAT4 gene and insulin resistance and diabetes risk, especially diabetes type I [23-25]. In our study we analysed the association between the STAT4 rs7574865 polymorphism and PTDM. Our results did not reveal any significant association between this polymorphism and PTDM.

Bolin et al. examined STAT4 polymorphisms in patients with lupus nephritis in two cohorts of Swedish patients. These authors have indicated that the STAT4 rs7582694 polymorphism may predispose to lupus nephritis and a worse outcome of this disease with severe renal insufficiency [26]. Only one study has examined the role of the STAT4 rs7574865 polymorphism in kidney allograft function. Yang et al. evaluated the association between the STAT4 rs7574865 polymorphism and acute allograft rejection in the Chinese population [27]. No evidence of an association was found between healthy controls and renal transplant recipients for the GT or TT genotype and the wild-type GG. However, among the transplant recipients, the GT or TT genotype was more common in patients with acute allograft rejection than in those without rejection who mostly had the wild-type GG genotype. Thus, the authors suggested that the STAT4 rs7574865 SNP is a genetic susceptibility variant for acute renal allograft rejection in the Chinese population.

Among our transplant recipients, there was no statistically significant association between the STAT4 rs7574865 SNP and acute allograft rejection. This lack of association may be caused by ethnic differences. Our patients were from a Caucasian population with different genetic backgrounds than the Chinese population. Our results suggest a lack of association between the STAT4 rs7574865 SNP and kidney allograft function in the Polish population. Nevertheless, the role of STAT4 in immunity and allograft function requires further investigation.

\section{References}

1. Turkson J (2004): STAT proteins as novel targets for cancer drug discovery. Expert Opin Ther Targets 8: 409-422.

2. Kaplan MH (2005): STAT4: a critical regulator of inflammation in vivo. Immunol Res 31: 231-242.

3. Levy DE, Darnell JE Jr (2002): Stats: transcriptional control and biological impact. Nat Rev Mol Cell Biol 3: 651-662.

4. O’Malley JT, Sehra S, Thieu VT, et al. (2009): Signal transducer and activator of transcription 4 limits the development of adaptive regulatory T cells. Immunology 127: 587-595.

5. Nakayamada S, Kanno Y, Takahashi H, et al. (2011): Early Th1 cell differentiation is marked by a Tfh cell-like transition. Immunity 35: 919-931.

6. Watford WT, Hissong BD, Bream JH, et al. (2004): Signaling by IL-12 and IL-23 and the immunoregulatory roles of STAT4. Immunol Rev 202: 139-156.

7. Bi C, Li B, Cheng Z, et al. (2013): Association study of STAT4 polymorphisms and type 1 diabetes in Northeastern Chinese Han population. Tissue Antigens 81: 137-140.
8. Remmers EF, Plenge RM, Lee AT, et al. (2007): STAT4 and the risk of rheumatoid arthritis and systemic lupus erythematosus. N Engl J Med 357: 977-986.

9. Liu QF, Li Y, Zhao QH, et al. (2015): Association of STAT4 rs7574865 polymorphism with susceptibility to inflammatory bowel disease: A systematic review and meta-analysis. Clin Res Hepatol Gastroenterol 39: 627-636.

10. Korman BD, Kastner DL, Gregersen PK, et al. (2008): STAT4: genetics, mechanisms, and implications for autoimmunity. Curr Allergy Asthma Rep 8: 398-403.

11. Korman BD, Alba MI, Le JM, et al. (2008): Variant form of STAT4 is associated with primary Sjögren's syndrome. Genes Immun 9: 267-270.

12. Lu X, Perkins DL (2005): A systems biology view of allograft rejection. Curr Opin Organ Transpl 10: 28-34.

13. Solez K, Colvin RB, Racusen LC, et al. (2008): Banff 07 classification of renal allograft pathology: updates and future directions. Am J Transplant 8: 753-760.

14. Davidson J, Wilkinson A, Dantal J, et al. (2003): New-onsetdiabetes after transplantation: 2003 International consensus guidelines. Proceedings of an international expert panel meeting. Barcelona, Spain, 19 February 2003. Transplantation 75: S3-24.

15. Huang Y, Lei Y, Zhang H, et al. (2011): MicroRNA reulation of STAT4 protein expression: rapid and sensitive modulation of IL-12 signaling in human natural killer cells. Blood 118: 6793-6802.

16. Chang JS, Wiemels JL, Chokkalingam AP, et al. (2010): Genetic polymorphisms in adaptive immunity genes and childhood acute lymphoblastic leukemia. Cancer Epidemiol Biomarkers Prev 19: 2152-2163.

17. Chang HC, Han L, Goswami R, et al. (2009): Impaired development of human Th1 cells in patients with deficient expression of STAT4. Blood 113: 5887-5890.

18. Liang Y, Christopher K, DeFina R, et al. (2003): Analysis of cytokine functions in graft rejection by gene expression profiles. Transplantation 76: 1749-1758.

19. Zhou P, Szot GL, Guo Z, et al. (2000): Role of STAT4 and STAT6 signaling in allograft rejection and CTLA4-Ig-mediated tolerance. J Immunol 165: 5580-5587.

20. Eurich D, Boas-Knoop S, Struecker B, et al. (2013): Genetic variants of STAT-4 affect the development of graft fibrosis after liver transplantation for $\mathrm{HCV}$-induced liver disease. Transplantation 95: 203-208.

21. Shuai K, Liu B (2003): Regulation of JAK-STAT signalling in the immune system. Nat Rev Immunol 3: 900-911.

22. Zervou MI, Mamoulakis D, Panierakis C, et al. (2008): STAT4: a risk factor for type 1 diabetes? Hum Immunol 69: 647-450.

23. Lee HS, Park H, Yang S, et al. (2008): STAT4 polymorphism is associated with early-onset type 1 diabetes, but not with late-onset type 1 diabetes. Ann N Y Acad Sci 1150: 93-98.

24. Yi J, Fang X, Wan Y, et al. (2015): STAT4 polymorphisms and diabetes risk: a meta-analysis with 18931 patients and 23833 controls. Int J Clin Exp Med 8: 3566-3572.

25. Li C, Zhao L, Wang W, et al. (2015): STAT4 rs7574865 polymorphism contributes to the risk of type 1 diabetes: a meta analysis. Int J Clin Exp Med 8: 2471-2475.

26. Bolin K, Sandling JK, Zickert A, et al. (2013): Association of STAT4 polymorphism with severe renal insufficiency in lupus nephritis. PLoS One 8: e84450.

27. Yang H, Zhou Q, Chen ZM, et al. (2011): Polymorphisms in STAT4 increase the risk of acute renal allograft rejection in the Chinese population. Transpl Immunol 24: 216-219. 